\title{
Statistical Regularization of Deformation Fields for Atlas-Based Segmentation of Bone Scintigraphy Images
}

\author{
Karl Sjöstrand, Mattias Ohlsson, and Lars Edenbrandt \\ EXINI Diagnostics AB, Lund, Sweden \\ karl.sjostrand@exini.com
}

\begin{abstract}
The construction and application of statistical models of deformations based on non-rigid image registration methods have gained recent popularity. This paper presents the application of such a model to restricting a general-purpose registration algorithm to anatomically plausible solutions. Specifically, the Morphon registration method is used for atlas-based segmentation of bone scintigraphy images. From a training set of 734 images, a model of characteristic deformation fields is built and used for regularizing the registration of 113 test images. Results show that around 300 training images and 30 principal modes are sufficient for building a useful model. The segmentation succeeded in 106 of 113 test images.
\end{abstract}

\section{Introduction}

Accurate segmentation of bone scintigraphy images is a prerequisite for localizing and quantifying skeletal metastatic disease. Disease extent is an important prognostic indicator of survival longevity [1]. Automating the segmentation and the subsequent chain of analysis may both increase effectiveness and objectivity of the clinical investigation. In this paper, we describe and apply a modified version of the Morphon [2] algorithm for aligning a segmented atlas image to patient images. The principal modification pertains to the regularization of the vector field of deformations obtained during each iteration. To guide the registration towards anatomically plausible solutions, the deformation field is constrained with respect to a statistical deformation model [3] (SDM) built from examples. Previous reports on such models regarded small sets of examples and high-dimensional volumetric data 34. Here, we focus on data of limited dimensionality and a relatively large training set to enable the investigation of the relations between model dimensionality, training set size, and generalization ability. The performance of the modified Morphon is measured from the segmentation of a test set of images.

The Morphon registration algorithm was devised by Knutsson and Andersson [2. Petterson et al. 5] present an application of the Morphon to the segmentation of the pelvis from CT images. The term and concept of SDMs are due to 
Rueckert et al. 3]. SDMs have been applied directly to registration by optimization in the space of principal components 6 6/7/4, for the automatic construction of shape models [8, and for interpretation [10].

\section{Method}

This section presents a review of the Morphon method for image registration along with proposed modifications. Where applicable, we follow the nomenclature of Knutsson et al 2. Image dimensionality is denoted by $p_{d}$ while $p$ is the number of image elements (pixels or voxels) and $n$ is the number of images (subjects). The images studied in this paper are two-dimensional $\left(p_{d}=2\right)$, however, the discussed methodology applies to images of arbitrary dimensionality.

The Morphon method follows an iterative scheme where local image deformations are used to deform a source image with the aim of bringing it into successively closer correspondence with a target image. Instead of directly optimizing an image similarity measure, each Morphon iteration attempts to mitigate differences in location on a per-image element basis, subject to constraints on the irregularity of the resulting deformation field; a process which implicitly increases image similarity in successful cases. Each iteration consists of four steps; deformation of the source image according to the current accumulated deformation field, estimation of a new deformation field, deformation field regularization, and the addition of the regularized deformation field to the accumulated field. Each of these steps will be described briefly below.

Image Deformation. Each iteration starts with the deformation of the source image according to the current accumulated field (see below). This deformation is carried out using standard image warping techniques.

Deformation Field Estimation. A new deformation field is estimated from measurements of local phase differences between the deformed source image and the target image. Image phase is estimated using complex filters sensitive to intensity ridges, valleys and edges in a particular direction. A set of $n_{f}$ filters (here, $n_{f}=4$ ) are created to cover equally spaced directions on the unit circle. The complex filter response of each filter is separated into phase and magnitude components. The phase difference between the deformed source and target images at a particular element is proportional to the displacement of the element in the source image relative the target image. The magnitude of the response provides a measure of the certainty of the displacement. Using the filter directions as predictor variables and the estimated phase difference as the response, the deformation vector corresponding to an image element can determined by weighted least squares,

$$
\arg \min _{\mathbf{v}} \sum_{i=1}^{n_{f}} c_{i}\left(\mathbf{n}_{i}^{T} \mathbf{v}-d_{i}\right)^{2}
$$


where $\mathbf{v}$ is the $p_{d} \times 1$ deformation vector to be estimated, $\mathbf{n}_{i}$ is the direction of the $i^{\text {th }}$ filter, and $d_{i}$ and $c_{i}$ are the ditto phase response difference and magnitude respectively. Solving this equation for all image elements yields an initial deformation field estimate. We propose to augment Equation 1 to enable the registration of multi-band images such as RGB color images and multi-spectral and multi-modality images, where several images are acquired simultaneously and in correspondence. Bone scintigraphy is such an image type, as there are two corresponding images per patient, one anterior and one posterior. In such cases, the task is to determine a single deformation field from multiple images. Equation 1 can be augmented to include the additional information provided by several images in the following manner,

$$
\arg \min _{\mathbf{v}} \sum_{j=1}^{n_{b}} \sum_{i=1}^{n_{f}} c_{i, j}\left(\mathbf{n}_{i, j}^{T} \mathbf{v}-d_{i, j}\right)^{2}
$$

where $n_{b}$ is the number of corresponding images (bands). This makes the size of the system $n_{f} n_{b} \times p_{d}$ instead of $n_{f} \times p_{d}$. The effect of this is that $\mathbf{v}$ can be determined based on more data, weighted by the certainty at each element, band and direction.

Deformation Field Regularization. The deformation field estimation neither models spatial dependencies among the deformation vectors nor enforces smoothness. Instead, this is incorporated in a subsequent regularization step on which much of this paper focuses. The Morphon method suggests a filtering approach known as normalized averaging resulting in elastic deformations 211. Each component matrix of the deformation field is convolved with a Gaussian kernel. To increase robustness, the certainties are included which allow more certain deformations to have higher influence on the averaging at each element. Let the matrix $\mathbf{d}$ denote a component (e.g. the $x$-values) of the deformation field. Then, the regularized deformation field component is obtained by,

$$
\mathbf{d}_{\text {reg }}^{\text {elastic }}=\frac{(\mathbf{c} \odot \mathbf{d}) * \mathbf{g}}{\mathbf{c} * \mathbf{g}},
$$

where $\mathbf{g}$ is a low-pass Gaussian filter kernel, * is a convolution operator and $\odot$ is the Hadamard (element-wise) product operator. This procedure is carried out separately for all components.

During the first stages of the registration process, it may be beneficial to regularize the deformations further. We do this by projecting the deformation coordinates onto an affine basis in a weighted least-squares sense using the certainties as weights.

Deformation Field Accumulation. The obtained regularized deformation field is added to the total deformation field describing the transform from the original source image to the target. The certainties influence this accumulation 
such that less certain deformations affect the the total field less than more certain counterparts. The accumulated field components $\mathbf{d}_{a}$ and the accumulated certainties $\mathbf{c}_{a}$ are updated in each iteration according to

$$
\mathbf{d}_{a}=\frac{\mathbf{c}_{a} \odot \mathbf{d}_{a}+\mathbf{c} \odot\left(\mathbf{d}_{a}+\mathbf{d}\right)}{\mathbf{c}_{a}+\mathbf{c}} \quad \text { and } \quad \mathbf{c}_{a}=\frac{\mathbf{c}_{a}^{2}+\mathbf{c}^{2}}{\mathbf{c}_{a}+\mathbf{c}} .
$$

We will now turn to a description of the proposed alternative to normalized averaging for displacement field regularization.

\subsection{Building a Model of Characteristic Deformations}

Using the described registration method we created an intensity-based atlas from ten bone scintigraphy images of normal subjects. The atlas was created as outlined in [12, where all subjects are registered to an arbitrary member of the normal group. The average deformation field is then calculated and used to transform all subjects to the common average anatomy. To decrease the bias from choosing a particular member as reference, the process is repeated with the estimated average anatomy and intensity as reference. The atlas was segmented into 31 anatomical regions by a medical expert. When registering an unknown bone image to this atlas, the atlas segmentation can be transformed using the inverse of the resulting deformation field, providing a segmentation of the unknown image.

We successfully registered 734 bone images to this atlas, resulting in equally many deformation fields. Affine transformation components were factored out, leaving a set of fields describing the non-rigid differences between each subject and the atlas. Each field was reshaped into a single row vector, e.g. as $\left[x_{1} \ldots x_{p} y_{1} \ldots y_{p}\right]$ and put in a mean centered data matrix $\mathbf{X}$ of size $n \times p_{d} p$. Performing a principal component analysis (PCA) on these data amounts to finding and orthogonal matrix $\mathbf{L}$ and a diagonal matrix $\mathbf{D}$ such that $n^{-1} \mathbf{X}^{T} \mathbf{X L}=\mathbf{L D}$. However, as $p \gg n$ for most SDMs, the computation of the $p_{d} p \times p_{d} p$ covariance matrix $n^{-1} \mathbf{X}^{T} \mathbf{X}$ becomes difficult. The principal components and their variances can instead be obtained by

$$
\mathbf{L}=\frac{1}{\sqrt{n}} \mathbf{X}^{T} \hat{\mathbf{L}} \hat{\mathbf{D}}^{-\frac{1}{2}}, \quad \mathbf{D}=\hat{\mathbf{D}}
$$

where $\hat{\mathbf{L}}$ and $\hat{\mathbf{D}}$ are the eigenvectors and eigenvalues respectively of the smaller $n \times n$ matrix $n^{-1} \mathbf{X} \mathbf{X}^{T}$.

The deformation fields are defined on a rectangular grid of which only part is occupied by the object of interest, the skeleton. The decomposition of the deformation field data can be done as suggested above, but much of the model dimensionality will be spent on describing deformations which occur outside the skeleton. This can be alleviated by performing an importance-weighted PCA [13]. We used the mean certainty map calculated from all 734 training images as weights and put them (repeated $p_{d}$ times) in a diagonal matrix $\mathbf{W}$. The decomposition can then be performed as above using the weighted data set $\tilde{\mathbf{X}}=\mathbf{X W}$. 


\subsection{Model-Based Regularization of Deformation Fields}

Regularization using normalized averaging is general in the sense that it allows any transformation with sufficient smoothness. Many such transformations lead to anatomically implausible solutions. Regularization can instead be provided by the model of characteristic transformations from Equation 5. The regularization consists of a projection of the obtained deformation field $\mathbf{d}$ (here represented by a length $p_{d} p$ column vector) onto a rank $k$ subspace by

$$
\mathbf{d}_{\text {reg }}^{\mathrm{SDM}}=\mathbf{W}^{-1} \mathbf{L}_{k} \mathbf{L}_{k}^{T} \mathbf{W}\left(\mathbf{d}-\mathbf{d}_{\text {affine }}\right)+\mathbf{d}_{\text {affine }},
$$

where $\mathbf{L}_{k}$ consists of the $k$ columns of $\mathbf{L}$ corresponding to the $k$ largest eigenvalues and $\mathbf{d}_{\text {affine }}$ is the affine component of $\mathbf{d}$.

\section{Results}

To determine whether the given number of training images is sufficient for building a model that is general enough for segmenting unseen images, we performed two studies. The first is a five-fold cross-validation study measuring the discrepancy between the "true" deformation fields as given by standard Morphon segmentation of the 734 training set cases and the model approximation of the corresponding fields. The error measure was $s^{-1} \sum_{i=1}^{s}\left\|\mathbf{d}_{i}-\mathbf{d}_{i(\mathrm{reg})}^{\text {SDM }}\right\|^{2}$ where $s=\lfloor 734 / 5\rfloor$ is the number of images in the test fold. This measure was evaluated for an increasing number of modes and for eight data set sizes; $n=50$, 148, 245, 343, 441, 539, 636 and 794. We also evaluated the measure without cross-validation, i.e. directly on the training data. Figure 1(a) shows the resulting eight cross-validation error curves and the training error curve. From a computational viewpoint, cross-validation is impractical for high-dimensional data sets; the study presented here took days to compute. A less demanding alternative is to, as is customary, assume that the relevant modes explain e.g. $95 \%$ of the total variation. Focusing on the eigenvalues of submatrices of the "small" covariance matrix, we plot the number of modes necessary to explain a certain fraction of the total variance given a data set size. Figure $1(\mathrm{~b})$ shows this plot for fractions $90 \%, 95 \%, 97 \%$ and $99 \%$. The model size saturate at around 30 modes and 300 training images. The cross-validation study also shows scant improvement for models built from more that 300 images, but seems to suggest a larger model (around 100 modes). Parallel analysis [14] performed on repetitions of permuted variables and all 734 images suggested 27 modes. Based on this information and favoring a more compact model, we chose a model of 30 modes built from all 734 images for the subsequent analysis.

Both the standard Morphon registration and the SDM-regularized registration were initiated with an affine alignment to account for gross differences. The SDM registration was then regularized using an increasing number of modes; $1,2,5,10$, and finally 30 . The algorithm was run for a fixed number of iterations (typically 5) for each of these regularization options. This proved sufficient for convergence. The SDM algorithm was run on a test set of 113 unique images/patients. Visual 


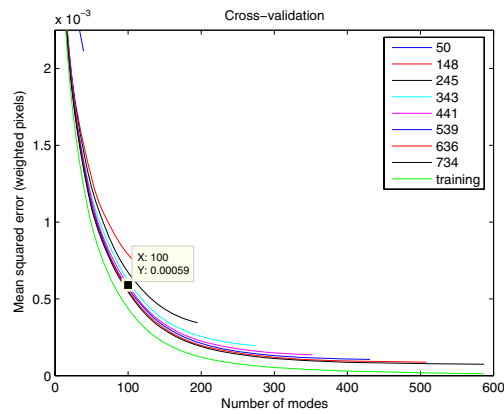

(a) 5-fold cross-validation

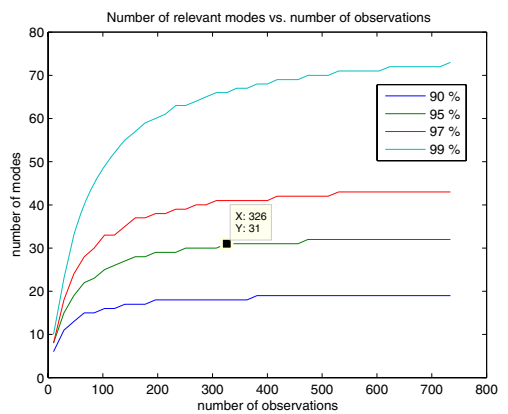

(b) Training set size versus suitable model size

Fig. 1. Curves for determining model dimensionality and the smallest training set size for which the addition of more training examples does not lead to a more general model. Both graphs indicate that models built from around 300 examples are sufficiently general, but cross-validation suggests models of higher dimensionality than the analysis of the principal component variances.

inspection yielded 7 failures, 6 with minor registration errors and 1 with more pronounced errors. The standard algorithm, which already had proven its value by succeeding in 734 of 795 training images, failed in 10 cases from the test set, 8 and 2 with minor and major errors respectively.

\section{Discussion}

Figure 2 shows the atlas with the manually defined segmentation superimposed along with examples of images where SDM regularization managed to guide the registration to a better segmentation than normalized averaging regularization. Example 1 shows a patient with a leg prosthesis which is not detected on scintigraphy images. SDM regularization leads to a more plausible segmentation in this region compared to standard regularization. The right arm is also better delineated with SDM regularization in this example. Example 2 shows an example where the skull segmentation was placed relatively far from from the actual skull after affine alignment. SDM regularization manages to preserve the skull shape and guide the segmentation into place while standard regularization leads to a smearing of the skull segmentation such that only part of the delineation is correct. The choice of incorporating a maximum of 30 modes for regularization was sufficient for obtaining accurate registrations on the test images. Raising this number to 100 as suggested by Figure 1(a) neither improved nor over-fitted the adjusted model to a significant extent.

The segmentation failure rates for SDM versus standard regularization suggests that performance is comparable between the methods. Often, the failures occurred for different patients, suggesting SDM regularization as a complement — rather than as a replacement — to normalized averaging. 


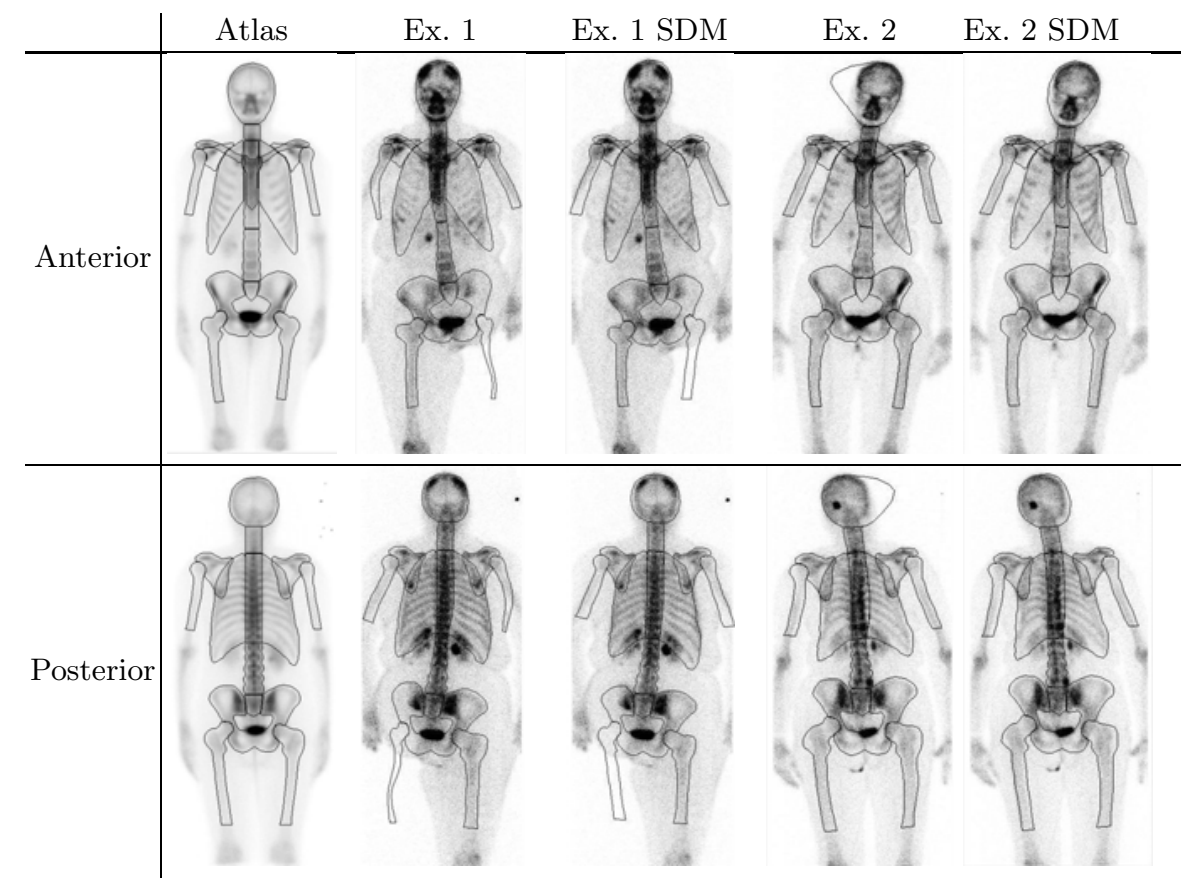

Fig. 2. Anterior and posterior bone scintigraphy images with the segmentation superimposed. The first column shows the atlas with the manually drawn segmentation. Columns 2 and 3 show example results from registering the atlas to a patient image using normalized averaging and SDM regularization respectively. Columns 4 and 5 show another example.

The Morphon registration method is based mainly on convolutions. Optimizing the algorithm for speed therefore amounts to creating an efficient convolution procedure. The registration of one patient containing one anterior and one posterior image took roughly 5-10 seconds in our Java implementation, for both normalized averaging and SDM regularization. The computational complexity for these regularization options is $\mathcal{O}(l p)$ and $\mathcal{O}(k p)$ respectively, where $l$ is the size of the (1-D) regularization kernel and $k$ is the number of modes.

The choice of decomposing the deformation data by an importance-weighted PCA had a large impact on model compactness. Performing a standard PCA lead to a model which required 73 modes to cover $95 \%$ of the variation while parallel analysis suggested 54 modes. The graphical method from Figure 1(b) suggested around 70 modes calculated from at least 500 training images, however, the curves did not flatten out as evidently as for the weighted decomposition. In general, our impression is that the permeating use of the certainty matrix in the Morphon algorithm makes a difference and sets it apart from other optical flow-type algorithms for registration.

In studies to come, we wish to investigate the possibility of using the described method for registering volumetric image data. Previous reports on SDMs have presented preliminary results on this $7 \mathbf{7 4}$. However, in light of the results 
presented here, the outlook of gathering enough data for building a sufficiently general model seems bleak for such complex data sets.

\section{References}

1. Miller, P.D., Eardley, I., Kirby, R.S.: Prostate specific antigen and bone scan correlation in the staging and monitoring of patients with prostatic cancer. British Journal of Urology 70, 295-298 (1992)

2. Knutsson, H., Andersson, M.: Morphons: Paint on priors and elastic canvas for segmentation and registration. In: Scandinavian Conference on Image Analysis, SCIA (2005)

3. Rueckert, D., Frangi, A.F., Schnabel, J.A.: Automatic construction of 3D statistical deformation models using non-rigid registration. IEEE Transactions on Medical Imaging 22, 77-84 (2003)

4. Thompson, S., Penney, G., Buie, D., Dasgupta, P., Hawkes, D.: Use of a CT statistical deformation model for multi-modal pelvic bone segmentation. In: SPIE Medical Imaging 2008: Image Processing, vol. 6914, pp. 9141-9141. SPIE (2008)

5. Pettersson, J., Knutsson, H., Borga, M.: Automatic hip bone segmentation using non-rigid registration. In: Proceedings of the IEEE International Conference on Pattern Recognition, Hong Kong, China (2006)

6. Loeckx, D., Maes, F., Suetens, P.: Temporal subtraction of thorax CR images using a statistical deformation model. IEEE Transactions on Medical Imaging 22, 1490-1504 (2003)

7. Wouters, J., D’Agostino, E., Maes, F., Vandermeulen, D., Suetens, P.: Non-rigid brain image registration using a statistical deformation model, vol. 6144., 614411. SPIE (2006)

8. Frangi, A.F., Rueckert, D., Schnabel, J., Niessen, W.J.: Automatic 3D ASM construction via atlas-based landmarking and volumetric elastic registration. In: Insana, M.F., Leahy, R.M. (eds.) IPMI 2001. LNCS, vol. 2082, pp. 78-91. Springer, Heidelberg (2001)

9. Heitz, G., Rohlfing, T., Maurer Jr., C.R.: Statistical shape model generation using nonrigid deformation of a template mesh. In: Fitzpatrick, J.M., Reinhardt, J.M. (eds.) Proceedings of the SPIE, Medical Imaging 2005: Image Processing, vol. 5747, pp. 1411-1421. SPIE (2005)

10. Ólafsdóttir, H., Hansen, M.S., Sjöstrand, K., Darvann, T.A., Hermann, N.V., Oubel, E., Ersbøll, B.K., Larsen, R., Frangi, A.F., Larsen, P., Perlyn, C.A., MorrissKay, G.M., Kreiborg, S.: Sparse statistical deformation model for the analysis of craniofacial malformations in the crouzon mouse. In: Ersbøll, B.K., Pedersen, K.S. (eds.) SCIA 2007. LNCS, vol. 4522, pp. 112-121. Springer, Heidelberg (2007)

11. Bro-Nielsen, M.: Medical Image Registration and Surgery Simulation. PhD thesis, Department of Mathematical Modelling, Technical University of Denmark (1996)

12. Guimond, A., Meunier, J.: Average brain models: A convergence study. Computer Vision and Image Understanding 77(77), 192-210 (2000)

13. Jolliffe, I.T.: Principal Component Analysis, 2nd edn. Springer, Heidelberg (2002)

14. Horn, J.L.: A rationale and a test for the number of factors in factor analysis. Psychometrika 30, 179-185 (1965) 